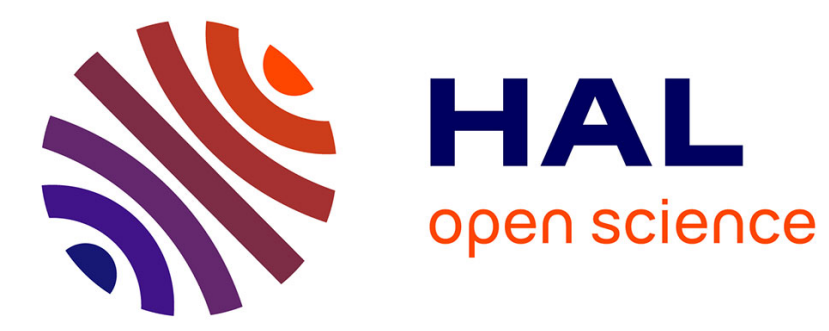

\title{
Contextualisations didactiques et didactologiques : questions en débat
}

\author{
Philippe Blanchet
}

\section{To cite this version:}

Philippe Blanchet. Contextualisations didactiques et didactologiques: questions en débat. Contextes et Didactiques, 2016, pp.8-14. hal-02518390

\section{HAL Id: hal-02518390 \\ https://hal.univ-rennes2.fr/hal-02518390}

Submitted on 25 Mar 2020

HAL is a multi-disciplinary open access archive for the deposit and dissemination of scientific research documents, whether they are published or not. The documents may come from teaching and research institutions in France or abroad, or from public or private research centers.
L'archive ouverte pluridisciplinaire HAL, est destinée au dépôt et à la diffusion de documents scientifiques de niveau recherche, publiés ou non, émanant des établissements d'enseignement et de recherche français ou étrangers, des laboratoires publics ou privés. 


\title{
Contextualisations didactiques et didactologiques : questions en débat
}

\author{
Philippe BLANCHET \\ PREFics $^{1}$ (EA 4246) - Université Rennes 2, Bretagne
}

\section{Résumé}

Cet article s'inscrit dans un débat concernant les notions-concepts de contexte et contextualisation. Après avoir rappelé l'élaboration conceptuelle proposée par l'auteur au cours de diverses publications parues ces dernières années, il examine des objections proposées non dans un cadre universaliste applicationniste mais, à l'inverse, du point de vue de la prise en compte de la diversité en didactique des langues. Il réfute ces objections et montre en quoi, tout en étant réaliste sur ses limites, une démarche de contextualisation est constructive en didactique et en didactologie des langues dans une perspective plurielle.

\section{Mots-clés}

Contexte, contextualisation, méthode, didactique, didactologie, sociodidactique, langues.

\begin{abstract}
This paper takes part into a debate about notions-concepts of context and contexualisation. After putting again how the author elaborated these concepts through various works these last years, it deals with objections from a linguistic diversity point of view rather than those from a universal applied linguistics point of view. It refutes the objections and shows that, although being realistic as far as its limits are concerned, a contextualising approach is helping for language teaching and language educations studies in a pluralistic perspective.
\end{abstract}

\section{Keywords}

Context, contextualisation, research and teaching method, language education, sociodidactics, languages.

\footnotetext{
${ }^{1}$ Laboratoire Plurilinguismes, Représentations, Expressions Francophones.
} 


\section{Introduction : passer de la notion au concept opératoire}

La notion de contexte est interprétable et utilisable de façon extrêmement variée, contradictoire, ambigüe. Il m'est apparu important (et on n'a pas manqué de m'interpeller très justement en ce sens), puisque j'en fais une question centrale (Blanchet, Moore et AsselahRahal, 2008/2009 ; Blanchet et Chardenet, 2011), définitoire d'une approche sociodidactique (Rispail et Blanchet, 2011), de passer de cette notion floue à un concept opératoire explicite et précisément défini, non seulement sur le plan théorique mais également sur le plan méthodologique. J'y ai donc consacré une série de séminaires pour l'élaborer, en discuter et le valider avec d'autres chercheur-e-s au cours des années 2012-2014. J'en ai rédigé la présentation en plusieurs versions, chacune prenant en compte les réactions à la version précédente (Blanchet, 2012a, 2012b, 2015). Je n'ajouterai pas ici une version de plus à ce texte (dont je considère pour l'instant la version 2015 suffisamment satisfaisante), mais je répondrai à certaines critiques générales faites contre l'usage de la notion en revenant sur sa nécessité et son caractère opératoire pour peu qu'on veuille bien examiner sa possible conceptualisation, par exemple celle que je propose ou bien sûr d'autres propositions.

Je crois toutefois utile de rappeler ici brièvement les éléments clés de la définition conceptuelle que j'ai proposés. Pour moi, le contexte doit être pensé comme une construction et non comme une donnée, car le contexte est doublement construit par rapport à la démarche de focalisation (ce sur quoi est centrée l'analyse et depuis quel point de vue) et par rapport au hors champ (le hors champ est ce qui n'est volontairement pas pris en compte, dont on ne fait pas un élément de contexte). Il me semble fondamental que le choix de ce qui constitue le contexte soit explicité dans la démarche de recherche et/ou d'intervention, notamment les critères selon lesquels on sélectionne consciemment ce qui fait contexte et ce qui reste hors contexte. J'insiste sur le fait qu'il doit s'agir, je crois, d'un processus réfléchi. Contextualiser ne se limite pas à "mettre en contexte », c'est-à-dire à (re)situer un phénomène ou un ensemble de phénomènes dans un contexte donné, qui lui reste extérieur, dont on l'a extrait et dans lequel on le réinsère éventuellement après observation. Contextualiser, c'est «mobiliser des éléments et des phénomènes qu'on choisit de faire entrer dans le champ d'observation au titre de contexte, c'est-à-dire de paramètres efficients mais qui ne sont pas au centre de la focale, pour comprendre les éléments et les phénomènes sur lesquels on focalise l'observation, phénomènes qu'on inscrit dans le continuum des pratiques sociales (car il s'agit pour moi d'étudier des pratiques sociales) ». En ce sens, le concept retrouve l'étymologie du mot qui sert à le désigner, puisqu'en latin, contextus signifie "assemblage », comme le rappellent opportunément Anciaux, Forissier et Prudent (2013 : 9), ainsi que Castellotti (2014 : 112) - mais sans pour autant que cette auteure y adhère.

\section{La contextualisation en didactique et en didactologie}

L'un des problèmes objectés à une démarche de contextualisation dans le champ didactique est dû à une certaine confusion entre intervention didactique et recherche didactologique. Si l'on prend par exemple deux études récentes qui tentent de faire des synthèses critiques (à mon avis erronées) des définitions et usages de la notion de contexte en didactique des langues (Castellotti, 2014 ; Debono et Pierozak, 2015), pour lancer un débat du point de vue la diversité, on y constate que les démarches de contextualisation examinées sont toutes des démarches d'intervention didactique ou orientées vers l'intervention didactique. Elles sont critiquées, à juste titre à mon avis malgré des amalgames abusifs (sur lesquels je reviendrai), comme étant des démarches d'adaptation plutôt simplistes, fondées sur des réifications des contextes, ne prenant pas assez en compte la question de la diversité. La démarche de 
contextualisation n'y est pas du tout (c'est explicite dans le texte de Debono et Pierozak) ou très peu (c'est flou dans le texte de Castellotti) examinée comme un point de méthode de recherche interprétative en didactologie. C'est négliger le fait que la notion de contexte et la démarche de contextualisation sont mobilisables dans les deux perspectives. En ce qui me concerne, c'est bien comme méthode de recherche que je l'ai élaborée, avec l'idée que des enseignant-e-s peuvent, à la façon des recherches-actions (Clerc, 2015), s'approprier cette méthode d'analyse interprétative pour élaborer de façon autonome des stratégies didactiques et pédagogiques qui contextualisent non pas de façon simpliste mais, au contraire, complexifiante (appropriation didactique de méthode de recherche déjà proposée dans Razafimandimbimanana et Blanchet, 2011, et qui se trouve au cœur des démarches de recherche-action).

Car dans un domaine comme le nôtre, cela a été dit et écrit des milliers de fois, on ne peut pas dissocier didactologie («recherche scientifique portant sur les pratiques d'élaborations et d'interventions didactiques ») et didactique ("pratiques d'élaborations et d'interventions d'enseignement-apprentissage »), même si l'usage courant et englobant de didactique comme hyperonyme de l'ensemble brouille regrettablement des polarités qui doivent être distinguées, mais non dissociées. Non seulement la didactologie porte sur la didactique, mais elle doit pouvoir proposer des orientations, des méthodes d'analyse, des outils... Non seulement la didactique nourrit mutuellement la didactologie, mais elle doit pouvoir lui proposer des pratiques, une réflexivité, des questionnements et des suggestions. L'enjeu social et humain des questions cruciales de formation, de langues, de relations, est déterminant. Je souscris en ce sens à la définition globale de la didactique des langues proposée par l'ACEDLE ${ }^{2}$ :

«Par didactique, nous entendons l'approche scientifique des processus d'enseignement et d'apprentissage des langues envisagés dans la diversité de leurs contextes. Cette approche se donne pour but et pour fonction sociale d'éclairer les partenaires impliqués dans l'acte éducatif sur les différentes stratégies possibles et sur les effets potentiels qu'ils sont en droit d'en attendre, de façon à rendre plus aisée leur prise de décision (...) Le décalage reste considérable entre l'univers de la recherche et celui de la conduite de classe au quotidien $»$.

\section{La question de la réification et du déterminisme}

Si l'on en croit Castellotti (2014), toutes les conceptions de la notion de contexte et de la démarche de contextualisation en didactologie sont réifiantes et « figées » au point « d'inhiber la réflexion » (Castellotti, $2014: 120)$ : elles poseraient toutes des contextes comme des "formes d'évidence et de transparence déterministes" (Castellotti, 2014: 121) avec notamment une vision simpliste de cultures comme des objets homogènes et stables (des " catégorisations solidifiées »; des « aspects fortement figés et stéréotypés ») qui sont utilisés pour analyser des situations de façon simpliste ou pour " au mieux adapter ou au pire appliquer » (Castellotti, $2014:$ 120) des outils didactiques dans de nouveaux « contextes » de façon tout aussi simpliste (d'où son invitation à "penser la diversité $\rangle^{3}$ qui présuppose que l'ensemble des chercheur-e-s et courants dont elle fait une revue rapide et partielle ne la «pensent» pas). C'est ce type de réification déterministe et simpliste (y compris dans leurs exagérations à prétention exhaustive) dont Debono et Pierozak (2015) font une analyse convaincante. Mais leur conclusion est, du coup, une invitation à se passer de la notion de

\footnotetext{
${ }^{2}$ Le Manifeste de l'Acedle disponible sur http://acedle.org/spip.php?article163.

${ }^{3}$ Castellotti (2014 : 121).
} 
contextualisation parce qu'elle est parfois (souvent selon eux) mal utilisée et ouvre des risques aussi grands qu'une démarche universaliste (Debono et Pierozak, 2014 : 45-46).

Je ne ferai pas ce pari pessimiste et je ne jetterai pas la baignoire à la place de l'eau d'un bain. Ce n'est pas parce que la «posture du juste milieu contextualisateur » (Debono et Pïerozak, 2014 : 44) est difficile à tenir, étant complexe au sens morinien du terme, ce n'est pas parce que des usages encore insatisfaisants de la contextualisation sont faits et sous ce nom, que la contextualisation est en soi et définitivement une pratique didactique à réfuter et exclure, et encore moins en tant que concept théorico-méthodologique opératoire en didactologie. Et il en va de même pour la contextualisation didactique au sens d' "adaptation » d'un enseignement à un contexte spécifique analysé de façon rigoureuse et explicite. D'autant qu'il est clair que Castellotti - aux yeux de qui personne ne trouve grâce dans son texte - y applique une généralisation abusive qui est tout le contraire de l'approche diversitaire qu'elle revendique et oppose, à tort, aux approches contextualisantes. Il suffit de lire les nombreux comptes rendus de recherches et d'interventions dans Blanchet et Martinez (2010), Feussi, Eyquem-Lebon, Moussirou-Mouyama et Blanchet (2010), Blanchet et Coste (2010), Blanchet et Chardenet (2011), Clerc (2011b), Kara, Kebbas et Daff (2013), Anciaux, Forissier et Prudent (2013), etc., ou tout simplement les deux exemples que je donne dans mon interview par Le français à l'université (Blanchet, 2009) pour vérifier l'attention de ces contextualisations didactologiques et didactiques à la diversité de situations individuelles et collectives qui ne sont pas réduites à des déterminismes généralisés ni à des stéréotypes. Il est donc possible, et bien sûr souhaitable, de contextualiser de façon souple, fine et pertinente, selon une démarche méthodique réfléchie.

\section{La question de l'autonomie didactologique par rapport aux disciplines contextualisantes}

Dernier point, la question du rapport à des disciplines contributives qui peuvent aider à contextualiser. L'une d'entre elles est particulièrement visée par les méfiances anticontextualisation, la sociolinguistique, parce qu'elle est au cœur de la question de la diversité linguistique. Ce n'est bien sûr pas la seule car, potentiellement, toute science humaine et sociale peut contribuer à comprendre les situations singulières d'individus et de groupes humains. Mais la sociolinguistique est, de façon cohérente, fortement mobilisée pour des contextualisations didactiques, au point qu'une approche contextualisante s'en soit en partie inspirée pour imaginer une sociodidactique en continuité avec une sociolinguistique (Rispail et Blanchet, 2011, pour une synthèse). L'idée est de tisser des liens au moins sur trois plans :

- 1. Pour élaborer une didactologie / didactique de quelque chose, il faut avoir une théorie de ce quelque chose (en l'occurrence une théorie des langues / pratiques linguistiques), et une théorie sociolinguistique est possible et même appropriée ;

- 2. Pour mobiliser et comprendre des éléments contextuels pertinents d'un enseignement-apprentissage des langues, une analyse sociolinguistique est possible et appropriée ;

- 3. Si l'on considère les situations de didactique des langues comme des situations sociolinguistiques, alors des méthodes de recherche sociolinguistiques sont possibles et appropriées. Cela ne signifie pas, bien sûr, qu'une approche didactologique et didactique en lien étroit avec une sociolinguistique soit la seule valable (ni bien sûr la seule utilisée), ce qui rend nécessaire de la nommer de façon claire, avec ce préfixe socio-, pour la distinguer d'autres approches moins ou non socio-. 
Castellotti (2014: 118) reproche à une telle approche d'être issue d'un "glissement de contexte à terrain » et d'être une " 'sociolinguistique appliquée' ou sociodidactique faisant découler directement les interventions didactiques de l'analyse des contextes/terrains sociolinguistiques », menée par des «sociolinguistes dans les projets et publications se réclamant de la $\mathrm{DDL}^{4} »$. On notera le discours qui consiste à borner un pré carré, celui où seuls les « vrais » didacticiens et didacticiennes seraient légitimes et dont on exclut ceux et celles avec qui on est en désaccord en leur assignant une identité étrangère, celle de « sociolinguistes ». Or ces arguments ne sont pas recevables au moins pour quatre raisons fondamentales. La première, c'est que l'enjeu est de travailler à un progrès des connaissances humaines et sociales, peu importe d'éventuelles frontières disciplinaires ou sousdisciplinaires, en grande partie arbitraires d'ailleurs, et d'autant que le champ didactologique a toujours été affirmé, plus que d'autres, comme bénéficiant d'une interdisciplinarité. La deuxième c'est qu'il ne s'agit pas d'un glissement impensé de contexte à terrain mais d'une reproblématisation de la notion de contexte en la confrontant à celle, problématisée aussi quoi qu'en dise Castellotti (2014: 118) ${ }^{5}$, de terrain (cf. Blanchet, 2012a : 31, par exemple). La troisième c'est que ce travail porte une attention particulière à la complexité et la diversité des procédures de transpositions qui ne sont pas exclusivement centrées sur les apports sociolinguistiques (Blanchet, 2011a). Enfin, la quatrième, c'est que les chercheur-e-s qui développent une approche sociodidactique se sont clairement inscrit-e-s dans le domaine didactologique tout au long de leur carrière, s'il était vraiment besoin de justifier leur identité professionnelle - qui peut être multiple (comme la mienne). La sociodidactique a été élaborée par des didacticien-ne-s soucieux de dimensions sociales et donc sociolinguistiques bien davantage que par des sociolinguistes d'origine investissant (à juste titre d'ailleurs le cas échéant) les terrains didactiques : qu'on pense à ces fondateurs-trices que sont dès les années 1980 C. Marcellesi, H. Romian, M. et L. Dabène, puis M. Rispail (1998, 2005), ainsi qu'aux travaux clés de S. Clerc (2011a), de C. Cortier (1998), d'A. di Meglio (1997) et de tous ceux et celles qui travaillent avec eux (cf. leurs publications collectives). Une sociodidactique n'est donc pas une sociolinguistique appliquée et les apports sociolinguistiques à une démarche de contextualisation ne disqualifient en rien cette démarche comme relevant du champ didactique et didactologique. On pourrait bien sûr en dire autant d'autres disciplines connexes et contributives, comme les sciences de l'éducation ou la sociologie (de l'éducation), ou encore la psychologie, par exemple.

\section{Conclusion : enjeux et limites de la contextualisation}

La perfection n'est pas atteignable et le perfectionnisme empêche d'agir. Ceci est vrai en toutes choses humaines et sociales, y compris en didactique et dans les tentatives de contextualisation. Il n'empêche qu'on peut tendre, du mieux possible, vers un progrès dans une direction dont on a la conviction raisonnée qu'elle est juste et utile au regard d'une éthique (pour moi d'une éthique humaniste altermondialiste comme je le dis dans Blanchet, 2009,2010 et 2011 b, entre autres). On peut à l'inverse paralyser l'action en renvoyant dos à dos, comme le font Castellotti (2014) et Debono et Pierozak (2015), un applicationnisme universaliste et une contextualisation qu'on estime insuffisante, y compris parce qu'on l'a examinée de façon trop rapide et trop globalisante. J'ai la conviction, à plusieurs titres, qu'il est efficace et urgent d'affirmer, de mettre en œuvre et de continuer à réfléchir une contextualisation humaine et sociale pour l'enseignement-apprentissage des langues.

\footnotetext{
${ }^{4}$ Didactique des langues.

5 «Les cultures, le terrain, les contextes, ne sont que très peu problématisés en tant que notions qui contribueraient alors à réfléchir les situations et à en proposer des interprétations ».
} 


\section{Références bibliographiques}

Anciaux, F., Forissier, T. et Prudent, L.-F. (dir.). (2013). Contextualisations didactiques. Approches théoriques. Paris : L'Harmattan.

Blanchet, P. (2009). Contextualisation didactique, de quoi parle-t-on ? Entretien avec Philippe Blanchet. Le Français à l'Université, 2, AUF. Disponible en ligne: http://www.bulletin.auf.org/IMG/pdf_Journal_AUF_14-2-3.pdf.

Blanchet, P. (2010). Post-face en forme de coup de gueule: pour une didactique de l'hétérogénéité linguistique - contre l'idéologie de l'enseignement normatif et ses discriminations glottophobes. Dans V. Feussi, M. Eyquem-Lebon, A. MoussirouMouyama et P. Blanchet (dir.), Hétérogénéité sociolinguistique et didactique du français. Contextes francophones plurilingues. Cahiers de Linguistique, 5(2), 165-183.

Blanchet, P. (2011a). Les transpositions didactiques. Dans P. Blanchet et P. Chardenet (dir.), Guide pour la recherche en didactique des langues et des cultures. Approches contextualisées (p. 97-201). Paris : Agence Universitaire de la Francophonie/Éditions des Archives Contemporaines.

Blanchet, P. (2011b). Quels fondements et quels objectifs pour une sociodidactique de la pluralité linguistique et culturelle? Enjeux épistémologiques, théoriques et interventionnistes. Dans S. Clerc (dir.) (Mé-)Tisser les langues à l'école ? Cahiers de Linguistique, 37(2), 183-192.

Blanchet, P. (2012a, [2000]). La linguistique de terrain, méthode et théorie. Une approche ethno-sociolinguistique de la complexité. Rennes : PUR.

Blanchet, P. (2012b). La contextualisation entre sociolinguistique et sociodidactique : enjeux théoriques et méthodologiques. Dans A.-Y. Abbes et M. Kara (dir.), Reconfiguration des concepts. Pour une réflexion épistémologique et méthodologique en sociolinguistique et sociodidactique. Revue SOCLES, 1, 13-20.

Blanchet, P. (2015). Contextes et contextualisations didactiques: définitions, enjeux théoriques, méthodologiques et interventionnistes. Dans P. Blanchet et P. Chardenet (dir.), Guide pour la recherche en didactique des langues et des cultures. Approches contextualisées. Édition revue et complétée (p. 107-110.). Paris : Agence Universitaire de la Francophonie / Éditions des Archives Contemporaines.

Blanchet, P. et Chardenet, P. (dir.) (2015 [2011]). Guide pour la recherche en didactique des langues et des cultures. Approches contextualisées. $2^{e}$ Édition revue et complétée. Paris : AUF / Éditions des Archives Contemporaines.

Blanchet, P. et Coste D. (dir.). (2010). Regards critiques sur la notion d'" interculturalité ». Pour une didactique de la pluralité linguistique et culturelle. Paris : L'Harmattan.

Blanchet, P. et Martinez, P. (dir.) (2010). Pratiques innovantes du plurilinguisme. Émergence et prise en compte en situations francophones. Paris : AUF/Archives Contemporaines.

Blanchet, P., Moore, D. et Asselah-Rahal, S. (dir.). (2009 [2008]). Perspectives pour une didactique des langues contextualisée. Paris: AUF/Éditions des archives contemporaines.

Castellotti, V. (2014). Contexte, contextualisation, cultures éducatives. Quels usages ? Pour quelles orientations de la recherche en DDL ? Dans S. Babault, M. Bento, L. Le Ferrec et V. Spaeth (dir.), Actes du colloque international Contexte global et contextes locaux. Tensions, convergences et enjeux en didactique des langues (p. 111-124). Paris: Université Paris Sorbonne/FIPF. Disponible en ligne sur http://www.academia.edu/12308739/Contexte_contextualisation_cultures_éducatives._ Quels_usages_Pour_quelles_orientations_de_- $1 \mathrm{a} \_$recherche_en_DDL_.

Clerc, S. (2011a). Vers une didactique $\bar{d}$ e la pluralité sociolinguistique. Synthèse d'Habilitation à Diriger des Recherches. Marseille : Université de Provence. 
Clerc, S. (dir.) (2011b). (Mé-)Tisser les langues à l'école ? Cahiers de Linguistique, 37(2).

Clerc, S. (2015). La recherche-action : ancrages épistémologique, méthodologique et éthique. Dans P. Blanchet et P. Chardenet (dir.), Guide pour la recherche en didactique des langues et des cultures. Approches contextualisées. Deuxième édition mise à jour et complétée (p. 111-121). Paris : AUF/EAC.

Cortier, C. (1998). Institution de l'Alliance française et émergence de la francophonie. Politiques linguistiques et éducatives (1880-1914). Thèse de doctorat non publiée, Lyon : Université Lyon 2.

Dabène, L. (1994). Repères sociolinguistiques pour l'enseignement des langues. Paris : Hachette.

Dabène, M. et Rispail, M. (2008). La sociodidactique : naissance et développement d'un courant au sein de la didactique du français en France. La Lettre de l'AIRDF, 42. 10-13.

Debono, M. et Pierozak, I. (2015). Enjeux et limites de la contextualisation en didactique diversitaire des langues-cultures. Réflexions en lien avec le projet Diffodia. Les cahiers de l'ACEDLE, 12(1), 23-53. Disponible en ligne sur: http://acedle.org/old/IMG/pdf/3_M-_Debono_et_I-_Pierozak.pdf.

Di Meglio, A. (1997). L'élaboration didactique d'une langue minorée : le corse. Problématique de l'enseignement du corse par l'approche sociolinguistique de la production de documents didactiques entre 1974 et 1994, Thèse de doctorat non publiée. Corte : Université de Corse.

Feussi, V., Eyquem-Lebon, M., Moussirou-Mouyama, A. et Blanchet, P. (dir.). (2010). Hétérogénéité sociolinguistique et didactique du français. Contextes francophones plurilingues. Cahiers de Linguistique, 5(2).

Kara, A.-Y., Kebbas, M. et Daff, M. (dir.). (2013). Dynamiques plurilingues : transpositions politiques et didactiques. Cahiers de Linguistique, 39(2).

Marcellesi, C., Romian, H. et Treignier, J. (1985). Quelques concepts et notions opératoires pour une pédagogie de la variation langagière. Repères, 67, 23-31.

Rispail, M. (1998). Pour une socio-didactique de l'oral en situation multiculturelle : le cas de l'oral. Thèse de doctorat non publiée. Grenoble : Université Grenoble 3.

Rispail, M. (2005). Plurilinguisme, Pratiques Langagières, Enseignement : pour une SocioDidactique des Langues. Synthèse d'Habilitation à Diriger des Recherches. Rennes : Université Rennes 2.

Rispail, M. et Blanchet, P. (2011). Principes transversaux pour une sociodidactique dite « de terrain ». Dans P. Blanchet et P. Chardenet (dir.), Guide pour la recherche en didactique des langues et des cultures. Approches contextualisées (p. 65-69). Montréal, Paris : Agence Universitaire de la Francophonie, Éditions des Archives Contemporaines.

Razafimandimbimana, E. et Blanchet, P. (2011). Enquêter sur le Plurilinguisme d'Enfants Migrants : Méthode et Enjeux au Regard d'un Terrain Québécois / An Investigation of the Multilingualism of Migrant Children: Method and Challenges in Quebec. Child and Health Education. 3(1), 31-46 [français], 47-61 [English]. Disponible en ligne sur: http://www.childhealthandeducation.com/articles/Abstracts/31Razab.htm. 